\title{
Gradhiva
}

GRADHIV

Revue d'anthropologie et d'histoire des arts

$6 \mid 2007$

Voir et reconnaître. L'objet du malentendu

\section{Yurupari ou les figures du diable}

Le quiproquo des regards croisés

Yurupari, the figures of the devil. The misunderstandings of the different points of view

\section{Dimitri Karadimas}

\section{OpenEdition}

Journals

Édition électronique

URL : http://journals.openedition.org/gradhiva/986

DOI : $10.4000 /$ gradhiva.986

ISSN : $1760-849 x$

\section{Éditeur}

Musée du quai Branly Jacques Chirac

Édition imprimée

Date de publication : 15 novembre 2007

Pagination : 44-57

ISBN : 978-2-915133-55-4

ISSN : 0764-8928

\section{Référence électronique}

Dimitri Karadimas, «Yurupari ou les figures du diable », Gradhiva [En ligne], 6 | 2007, mis en ligne le 15 novembre 2010, consulté le 01 mai 2019. URL : http://journals.openedition.org/gradhiva/986 ; DOI : 10.4000/gradhiva.986

Ce document a été généré automatiquement le 1 mai 2019.

(c) musée du quai Branly 


\section{Yurupari ou les figures du diable}

Le quiproquo des regards croisés

Yurupari, the figures of the devil. The misunderstandings of the different points of view

\section{Dimitri Karadimas}

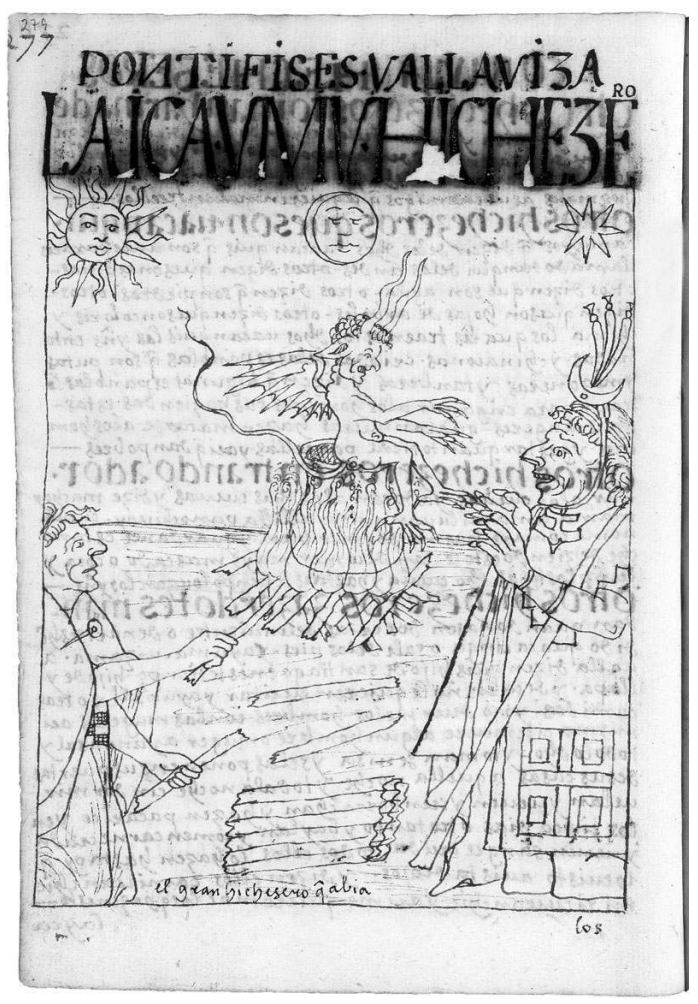

Fig. 1 Dessin datant de 1615 de l'Indien lettré Goaman Poma figurant le diable (associé à un prêtre inca), résultat de l'utilisation de la figure du diable et de son iconographie par les autorités ecclésiastiques à des fins de diabolisation des croyances locales (ici des régions andines).

Site de la Bibliothèque royale, université de Copenhague (2007). 


\section{Yurupari est-il le diable?}

Personnage énigmatique des traditions amérindiennes du Nord-Ouest amazonien depuis maintenant plus de trois siècles, Yurupari a été désigné et identifié comme le « diable » amérindien tant par les missionnaires qui tentaient de convertir ces populations que par les Indiens eux-mêmes -ce qui est plus surprenant.

2 L'attribution d'une qualité « diabolique » à des figures de croyances locales n'est pas un fait isolé dans les méthodes de conversion mises en œuvre par les missionnaires. Il ne s'agirait là, en fin de compte, que d'un cas parmi d'autres de procédures d'accusation permettant de destituer des religions concurrentes pour leur offrir le substitut de la croyance chrétienne. Mais pourquoi vouloir revenir ici sur ce qui est par ailleurs relativement connu?

3 L'une des raisons du relatif succès de ce complexe rituel et mythique au sein de la littérature amazonienne tient peut-être au fait que la première identification entre le diable et Yurupari ne semble pas entièrement l'œuvre des missionnaires mais plutôt celle des Indiens eux-mêmes. Si les Indiens du Nord-Ouest amazonien possédaient un système de croyances associé à une figure unique -même négative puisque liée au diable-, ils auraient été, aux yeux de ceux qui venaient les évangéliser, plus aptes que d'autres à recevoir le message de la foi chrétienne. Mais surtout, pour ces forces missionnaires, la trace de la présence divine des origines, bien qu'apparaissant comme dégénérée, se retrouverait chez Yurupari. Bref, d'une façon ou d'une autre, Yurupariétait la figure opposée mais complémentaire et nécessaire de Tupã, un équivalent du dieu créateur des chrétiens, créé par ces mêmes missionnaires.

4 Ce processus d'assimilation des héros civilisateurs au diable est toujours en vigueur, notamment chez les Curripaco (Arawak du haut río Negro) étudiés par Journet (1995). Ainsi, Kuai -équivalent de Yurupari dans cette tradition- est considéré, cette fois-ci par les missionnaires évangélistes, comme un personnage diabolique. Journet souligne cependant la contradiction de cette assimilation, puisque dans les termes mêmes de la mythologie curripaco il « est aussi, selon le mythe, le fils de Iapirikuri (l'équivalent du Créateur) et occupe donc la place du Christ dans l'évangile» (ibid.: 39). Cette contradiction apparaissait déjà dans le même groupe (les Wakuénai, autre appellation des Curripaco) au sein des mouvements millénaristes de la fin du XIXe siècle étudiés par Wright et Hill (1986) : Kuwai meurt dans un brasier (cf. infra) assimilé à l'enfer chrétien mais, en tant que héros civilisateur, il est en même temps assimilé au Christ. Les deux auteurs soulignent cependant la propension des leaders de ces mouvements à ne retenir que les éléments du symbolisme chrétien qui entrent en résonance avec ceux des cultures arawak de la région (ibid. : 33).

5 Mon propos n'est pas de remettre en question l'ensemble des connaissances accumulées depuis maintenant plus d'un siècle par la littérature ethnographique et ethnologique, voire, parfois, par les folkloristes, ni d'ailleurs d'en proposer une synthèse ${ }^{1}$. Je voudrais ici revenir sur cette première "reconnaissance » de la figure du diable dans celle de Yurupari pour interroger à la fois les mécanismes de la reconnaissance et ceux de la nomination. Mécanismes à l'œuvre, non pas tant dans un seul système culturel donné, mais, ce qui est plus communément le cas, lorsque les situations d'emprunts mutuels entre les sociétés donnent lieu à des quiproquos interprétatifs qui débouchent sur des 
cristallisations d'attribution d'altérité. Il s'agit ici de montrer que la «reconnaissance » ne fut pas tant le fait des missionnaires face à une figure de croyance locale qu'il fallait destituer, mais bien plus celui des populations indiennes qui, pour donner sens à la figure du diable véhiculée par les missionnaires et les colons, eurent recours à celle de leur propre panthéon avec laquelle elle partageait des traits formels. Ce n'est que par la suite que cette figure locale fut qualifiée de « Yurupari »par les missionnaires.

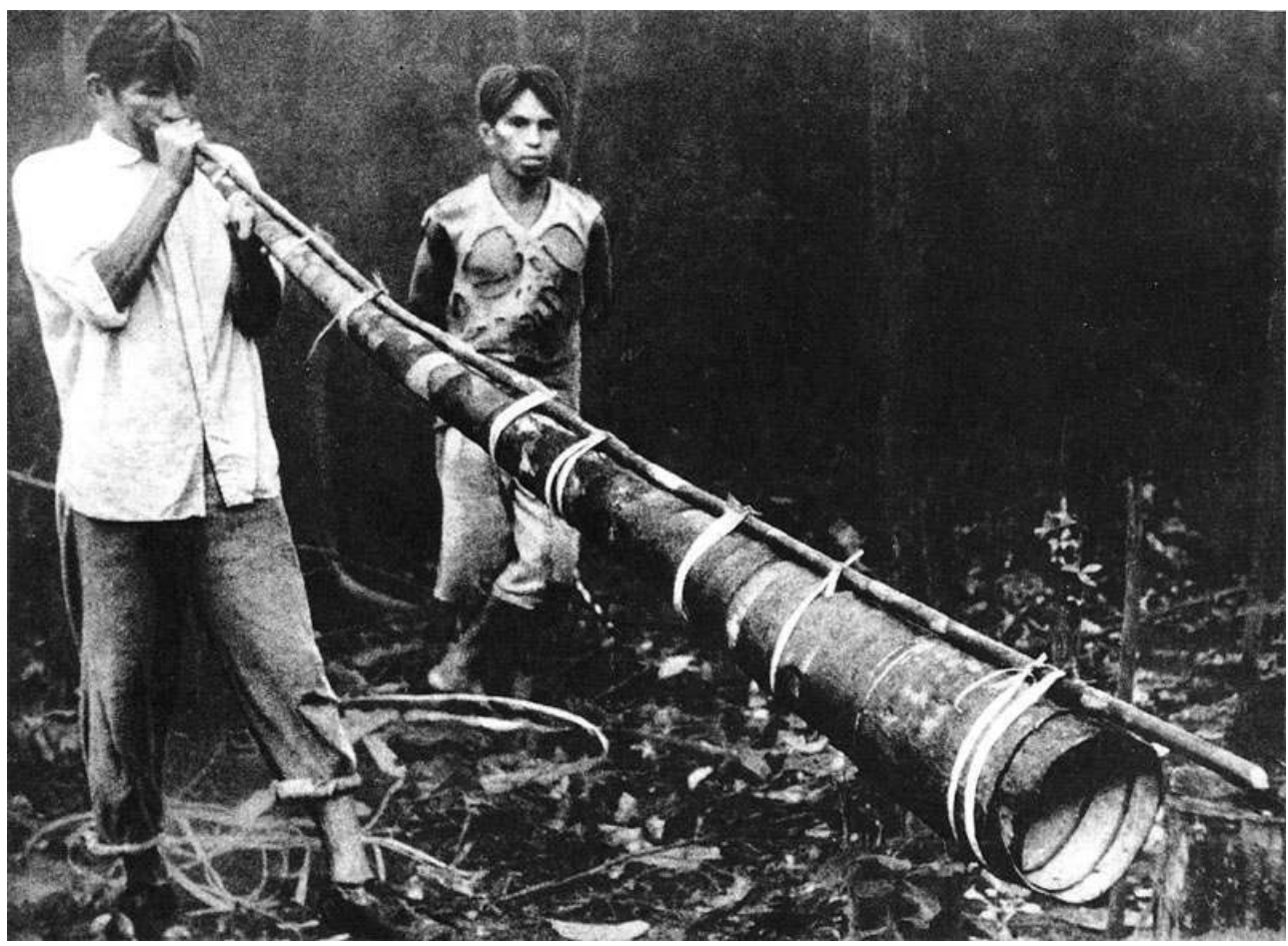

Fig. 2 Trompe en écorce enroulée, en usage dans plusieurs ethnies amazoniennes (Indiens ticuna, Brésil).

In Luis Antonio Escobar, La Musique précolombienne. Fondation Université Centrale, Intergráficas, Bogota, 1985.

\section{Les hypothèses existantes}

6 La littérature ethnographique à propos de Yurupari est abondante mais relativement récente $^{2}$. Parmi les dernières publications en date, on compte également celles des Indiens eux-mêmes ${ }^{3}$. À cela s'ajoutent de nombreux récits folkloriques ou d'inspiration littéraire (Orjuela 1983); cependant, ce sont les comptes rendus missionnaires, bien antérieurs, qui sont à l'origine de l'ensemble de la discussion.

Le terme en lui-même posséderait plusieurs origines : selon le père Tastevin, il signifierait «masque du visage» en ñengatú ou lingua geral, une lingua franca créée par les missionnaires à partir d'un dialecte tupi (ibid.). Il serait associé aux masques qui personnifient les esprits des animaux et/ou leur maître dans le Nord-Ouest amazonien. Les étymologies sont toutefois de peu de secours pour la compréhension du complexe rituel et du personnage éponyme. En effet, le qualificatif «Yurupari» fut assez systématiquement appliqué à toute figure qui, aux yeux des missionnaires, pouvait apparaître comme maléfique ou négative. Selon Orjuela, les premières hypothèses qui feraient correspondre Yurupari au diable proviendraient des missionnaires jésuites ayant 
associé une figure locale au "diable» tupi; pourtant, ce dernier serait également d'origine chrétienne (ibid. : 37).

8 La première description connue du rituel est proposée en 1689 par le père Fritz, jésuite d'origine néerlandaise (Cabrera Becerra 2002: 85), alors qu'il réside chez les Yurimagua, un groupe éloigné de la région du Vaupés. D'après ses descriptions, le groupe est situé de l'autre côté de l'Amazone (peut-être à l'embouchure du río Negro, mais la localisation n'est pas précise, pas plus que ne l'est l'identification du groupe ${ }^{4}$ ). Il est relativement facile de reconnaître, dans le tableau qu'il en dresse, une variante des rituels désignés aujourd'hui sous le terme yurupari dans le Nord-Ouest amazonien. Lorsque, dans son récit, le père Fritz s'inquiète de savoir d'où provient le son des trompes que l'on entend au loin, l'Indien lui révèle qu'il s'agirait du « diable » (Fritz 1997: 81). Il ajoute que ce dernier est aussi un représentant des bois et des animaux qui y résident et qu'il vient souvent sous leurs traits. Il est hélas impossible de reconnaitre, dans ce compte rendu des propos de l'Indien, s'il est fait ou non référence à une forme masquée.

Or, les populations amérindiennes de cette zone avaient déjà subi, avant toute présence missionnaire, plus d'un siècle de colonisation marchande (Reeve 1994 : 109). Fréquentes à l'époque, la figure du diable et ses représentations iconographiques ont probablement joué un rôle important dans les interactions entre les populations amérindiennes et les colons. Cette présence coloniale d'une figure religieuse chrétienne expliquerait l'identification « diabolique » proposée au père Fritz. À titre de comparaison, nous savons que le diable, non seulement par son évocation rhétorique, mais aussi par l'image, était couramment mis à contribution pour effrayer les populations indigènes, tels les Guarani au Xvire siècle (Martini 1990). Ainsi, Martini relate que les iconographies jouaient un rôle certain pour engendrer la peur. On utilisait le plus souvent des images issues de l'univers strictement chrétien en empruntant parfois également des figures du bestiaire local comme le jaguar (ibid. : 339). Or, l'utilisation de cette dernière figure n'est pas anodine pour le cas guarani. Comme Fausto l'a montré récemment (2007), les Guarani attribuant au chaman les qualités anthropophages du félin, lorsque l'on diabolise le jaguar, le chaman s'en trouve détrôné, menant, à terme, à une « dé-jaguarification »-c'est le terme même de Fausto- de l'ethos guarani (ibid.).

Ces terres de mission, certes éloignées du Nord-Ouest amazonien, étaient toutefois, comme ce dernier avant l'arrivée des moines capucins, sous la responsabilité catéchistique des jésuites. Ainsi, dès le début du xvire siècle, dans les Andes, plusieurs images figurant un diable relativement fidèle aux canons iconographiques chrétiens apparaissent dans les écrits de l'Indien lettré Goaman Pona (fig. 1). L'iconographie chrétienne proposée à des fins d'évangélisation comprenait donc les deux faces de sa dichotomie classique : aux côtés de la Vierge, de Jésus et des anges, figuraient en bonne place le diable et les enfers. Nul doute que ces iconographies furent l'autre visage que prirent les entreprises d'évangélisation, suivant un catéchisme qui n'hésita pas à vouloir enseigner tout en effrayant, et en assimilant figures de croyances locales et démonologie (voir Fleck 1999).

11 La question qui se pose, tant d'ordre ethnohistorique que relative aux systèmes de représentation, est donc de savoir comment les populations du Nord-Ouest au sens large ont pu interpréter ces iconographies, ou encore: qu'ont-elles pu voir dans ces images? Quel personnage auraient-elles été en mesure de reconnaître dans la figure du diable, et pour quelles raisons? 


\section{Les mythologies de Yurupari}

12 Le complexe de Yurupari englobe un ensemble de rituels et de mythes dans le Nord-Ouest amazonien, en partant des groupes appartenant à la famille arawak au nord-est, jusqu'aux Miraña et Huitoto au sud-ouest -mais il s'agit dans ce cas d'une mythologie différente-, voire aux Yurimagua du $\mathrm{xvII}^{\mathrm{e}}$ siècle à l'extrême sud, en passant par les groupes de langues tukano qui constituent le centre géographique de cet ensemble. Les rituels lors desquels les initiés sont mis en présence des flûtes et des trompes servent avant tout de rites d'initiation. Sans appartenir véritablement à une classe d'âge, de jeunes adolescents masculins sont arrivés à un stade de leur existence où ils doivent être séparés de la sphère féminine de la société. Ces flûtes et trompes sont interdites à la vue des femmes et des enfants. Lorsque le son des instruments retentit, les femmes doivent fuir la maison communautaire, accompagnées des non-initiés, ou se réfugier en son sein derrière un écran les maintenant à l'écart de la sphère publique. On ne devrait ainsi retenir sous le terme yurupari que les institutions indiennes qui interdisent aux femmes et aux jeunes enfants de voir les larges flûtes et trompes au son assourdissant, sous peine de châtiment. Si une femme en âge de procréer devait apercevoir l'un de ces instruments, elle serait mise à mort par empoisonnement (bien qu'à ma connaissance, un tel cas n'ait jamais été rapporté dans la littérature) ou encore violée. En d'autres termes, le rituel agit collectivement à la manière d'une menace proférée par les hommes vis-à-vis des femmes, de façon à asseoir sur elles leur autorité. Pour certains groupes du Vaupés, jusqu'au XIx ${ }^{e}$ siècle, il semble qu'un masque ait été associé au rituel et qu'il fût également interdit à la vision des femmes (fig. 3).

13 Dans la scène décrite par la figure 3, un crucifix est mis en parallèle avec un masque figurant Yurupari, présenté aux femmes tariana par le frère franciscain Coppi au XIX siècle $^{5}$. Cet épisode provoqua l'expulsion des franciscains de la localité (Wright 1992). Ce dessin permet de reconnaitre une cagoule surmontée de quelques plumes mais n'en offre pas une représentation exacte. Il semble que ce masque corresponde en partie à ceux qui sont confectionnés dans le Nord-Ouest amazonien à l'occasion de la fête des esprits des animaux (chez les Miraña, par exemple), ou pour d'autres fêtes faisant intervenir des êtres de la forêt, voire des ancêtres ${ }^{6}$. Or, les groupes du Vaupés qui utilisent de tels masques l'associent à leur héros civilisateur -désigné en lingua geral comme "Yurupari »et le représentent aujourd'hui avec un visage grimaçant associé à un large phallus en bois (pour une iconographie détaillée, voir Koch-Grünberg 1995 [1909]).

14 Il est donc possible que le personnage nommé «Yurupari » ait été soit figuré par un masque, soit incarné par de larges flûtes et trompes, laissant penser que deux réalités ou deux références mythico-rituelles ont été assimilées sous un même nom. Or il n'en est rien: c'est le même personnage qui se matérialise dans des artefacts distincts, chacun intervenant dans un rituel différent (les trompes lors des initiations, le masque pendant les fêtes des esprits des animaux au moment des fructifications).

Lors du rituel d'initiation, les jeunes sont fouettés, parfois jusqu'au sang, avec des verges faites de jeunes pousses d'arbre ou de lanières de cuir, avant que l'ensemble des hommes ne renouvellent entre eux ces flagellations. Chez les Barasana, ces instruments sont dénommés « dards de fourmis tocandira [Paraponera clavata] 》(Hugh-Jones 1979: 206). Ils rappellent -au moins sémantiquement- ceux utilisés lors des rituels d'initiation masculine dans les Guyanes, où des canevas de feuilles de palmier agrémentés de 
plusieurs dizaines de ces hyménoptères (guêpes et fourmis) sont appliqués sur le corps des initiés (Césard 2005).

Sans vouloir entrer dans le détail des différentes narrations mythiques ${ }^{7}$, il convient toutefois de revenir brièvement sur certains des épisodes clés de cet ensemble de mythes. Les principales sources ici considérées sont les travaux de Stephen Hugh-Jones sur les Barasana (1979), de Reichel-Dolmatoff sur les Desana et les Tukano (1996), la traduction en espagnol proposée par Orjuela (1983) de la version de Stradelli (la première à présenter, en 1890, une narration complète) et la récente ethnographie makuna proposée par Århem et al. (2004). J'ai recours, ici et là, à des informations présentées dans les écrits de Koch-Grünberg (1995 [1909]) et de Goldman (1963). Tous sont ethnographes -hormis Orjuela- de groupes ethniques appartenant au groupe linguistique tukano oriental.

Les différentes versions des mythes relatifs à Yurupariprésentent un personnage grandissant seul après avoir été séparé de sa mère (que certaines versions présentent comme vierge) à la naissance. Il est cependant doté d'une force et de pouvoirs extraordinaires, comme celui d'émettre de la lumière de son propre corps ou encore de produire du son par les nombreux orifices qu'il possède en plus de sa bouche, laquelle a dû lui être « ouverte » puisqu'il en était dépourvu. Certaines versions en font l'envoyé du Soleil en tant qu'entité céleste omnipotente, d'autres le présentent comme Tonnerre, ou son fils; quelques-unes l'identifient à une guêpe solitaire de couleur métallique. Il apparaît aussi sous la forme d'un anaconda (ou de son fils). Ces éléments ne sont pas contradictoires entre eux, mais se réfèrent aux différents états d'un même être subissant une série de métamorphoses, chacune étant décrite par un référent imagé (Tonnerre, par exemple, est le vrombissement produit par les ailes de la guêpe, voir Karadimas à paraître).

Toutes les versions s'accordent pour reconnaître à Yurupari une existence terrestre parmi les hommes. Il représente pour ceux-ci une figure majeure, dotée d'un rôle civilisateur, imposant une série de préceptes et d'interdits auxquels les humains devront se plier. Dans le mythe toujours, il dévore en guise de punition de jeunes garçons ayant transgressé l'interdit qu'il avait imposé sur la consommation de certains fruits (métamorphosé en caverne, d'après certaines versions, il se livre à un engloutissement anal de ces jeunes). Invité à un rituel par les parents qui veulent venger leurs morts, Yurupari ne tente pas de fuir devant le sort qui l'attend. Il l'accepte en connaissant par avance sa renaissance sous la forme du palmier paxiuba -socratea exorrhiza. Poussé dans un grand brasier, il se consume et le palmier dont on tirera les flûtes du rituel naît de ses cendres. Une fois les troncs sectionnés et les instruments confectionnés, les femmes s'approprient en premier les flûtes et les placent dans leur entrejambe ${ }^{8}$ : les hommes se voient alors obligés d'accomplir des tâches féminines. Yurupari intervient de nouveau en permettant aux hommes de récupérer les flûtes et oblige les femmes à porter la descendance des hommes en leur sein tout en leur interdisant, tant qu'elles sont fécondes, la vue des flûtes. 


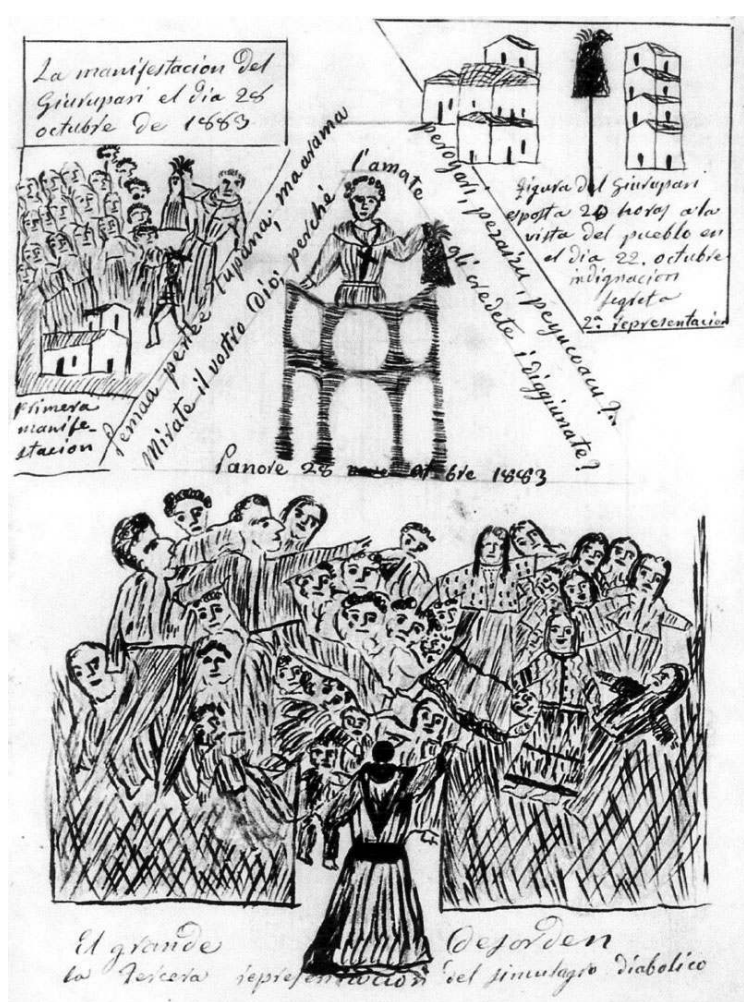

Fig. 3 Dessin réalisé par le frère franciscain Matteu Cagnari suite à la présentation par le frère F.G. Coppi d'un masque de « Jurupari » aux femmes indigènes tariana (Vaupés) en 1883. Interdit à la vue des femmes, le masque du personnage de la mythologie fut mis en parallèle avec un crucifix en demandant « qui était le vrai dieu ». Suite à cet épisode, les Tariana expulsèrent les religieux de la localité.

In Wright $1992: 261$

\section{Identification et reconnaissance}

Sans vouloir entrer dans les implications des différentes versions (voir Karadimas à paraitre), il faut toutefois revenir sur l'identification du personnage Yurupari comme guêpe solitaire de couleur métallique. Si j'insiste sur cette identification, c'est qu'elle me semble être un des éléments de compréhension du complexe rituel et des images auxquelles celui-ci fait appel. C'est aussi un indice permettant d'interpréter l'association entre l'iconographie des masques actuels des Miraña représentant le Maître des animaux à la fois avec l'espèce qui le personnifie et avec Yurupari. Il semble en effet que la guêpe solitaire fasse le lien entre les deux ensembles culturels -celui du Caquetá et celui du Vaupés- dans lesquels intervient cette figure mythologique. La guêpe est donc, ici et là, au centre des croyances liées à des personnages distincts de la mythologie. 


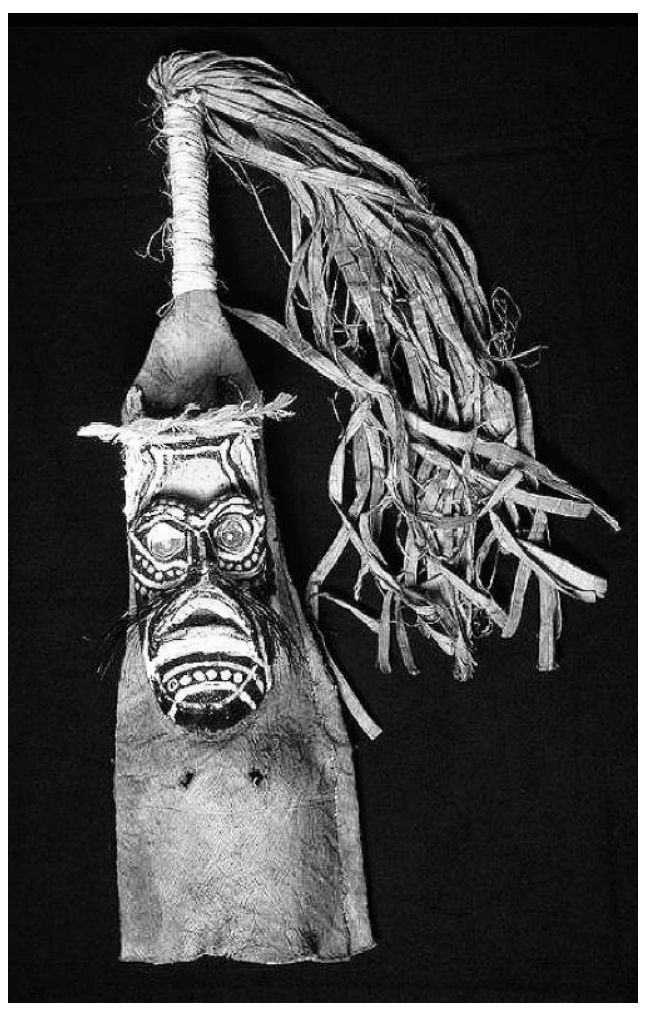

Fig. 4 Masque miraña représentant le personnage du Maître des animaux, lui-même une personnification d'une guêpe : on notera les deux « cornes » qui évoquent, une fois

anthropomorphisées, les mandibules de l'hyménoptère. Rituel des esprits des animaux, 1993, moyen Caquetá.

Photo Dimitri Karadimas.

Le mode de reproduction reconnu à cet hyménoptère le qualifie aux yeux des sociétés du Nord-Ouest amazonien pour en faire un personnage central dans la mythologie et dans le rituel. La femelle doit paralyser un insecte dans lequel elle pondra un œuf ou qu'elle apportera à une de ses larves déposées dans une cellule confectionnée avec de la boue (guêpes dites «maçonnes »). Le point crucial de cette identification est que les sociétés du Nord-Ouest amazonien interprètent ce comportement comme celui d'un mâle et non d'une femelle. L'insecte parasité joue le rôle de "mère" pour la descendance. Plus simplement, ce "mâle" se reproduit sans femelle et autorise ainsi une identification rituelle et un mode de constitution des adultes masculins sans référence aucune aux affins et à la partie féminine de la société (Karadimas 2003b, 2005, à paraitre).

Cette attribution de qualités masculines à une femelle est principalement due à une projection anthropomorphique -une des opérations majeures de connaissance de l'esprit humain (Karadimas 2005)- qui fait de l'abdomen de l'insecte un pénis, et du dard une forme de sperme, véhicule de la larve, c'est-à-dire de l'enfant à naître. Le rituel d'initiation mobilise une figure qui incarne la masculinité belliqueuse, à laquelle les initiés sont invités à s'identifier et que les femmes se doivent de craindre. Rappelons que, chez les Barasana, les initiés sont fouettés avec un instrument nommé " dard de fourmi ", c'est-à-dire qu'ils sont partiellement féminisés durant cette phase rituelle. C'est le même comportement dominateur, dont ils sont les victimes, que les hommes devront adopter vis-à-vis de la gent féminine une fois leur initiation accomplie. Le son des flûtes et des trompes est produit pour rappeler le bourdonnement de l'hyménoptère qui chasse ses proies (ses «femmes»), raison pour laquelle les femmes doivent fuir la maison 
communautaire lorsqu'elles les entendent, à l'image des araignées qui se cachent lorsqu'elles captent le vrombissement produit par leur prédateur (Karadimas à paraître). L'identification de la guêpe femelle à un mâle, qui réussit à se reproduire sans l'aide d'une femelle de sa propre espèce, constitue un modèle qui est également figuré chez les Miraña par un costume-masque doté d'un phallus en bois représentant le dard de l'insecte ${ }^{9}$ (fig. 5).

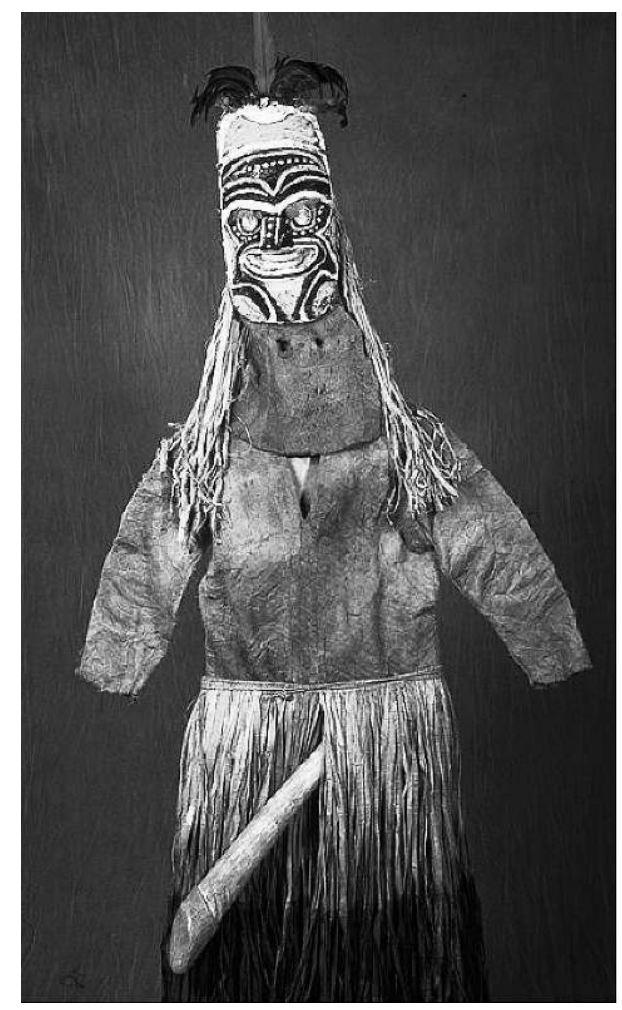

Fig. 5 Costume masque miraña représentant le Maître des animaux. Anthropomorphisation d'une guêpe parasitoïde, le phallus représente le dard de l'insecte. Rituel des esprits des animaux, 1993, moyen Caquetá.

Photo Dimitri Karadimas.

Chez les Miraña, une des modalités d'exécution du masque du Maître des animaux est de le doter de deux petites excroissances (fig. 4) qui, situées en haut de sa tête, forment des «cornes». Pourtant, ces excroissances ne représentent pas des cornes, mais les mandibules de l'hyménoptère qui, anthropomorphisées, c'est-à-dire mises "debout ", sont alors dirigées vers le haut. Placée de la sorte, la bouche est localisée en haut de la tête du masque qui se trouverait, de fait, "sans bouche", selon une perception frontale cette fois-ci puisqu'il est porté par un danseur. Le masque reçoit donc une bouche supplémentaire, correspondant à son caractère anthropomorphique, mais n'en perd pas pour autant ses deux excroissances. Celles-ci sont qualifiées de "cornes", uniquement cependant aux yeux d'une tradition iconographique qui en posséderait, comme celle qui en attribue au diable. Étant aujourd'hui familiers des représentations iconographiques du diable, certains Miraña nomment cette figure el diablito en espagnol, en fonction des similitudes formelles que les deux figures partagent, «cornes» comprises, sans pour autant l'assimiler au personnage de la tradition chrétienne.

Theodor Koch-Grünberg décrit dans son ethnographie de certains groupes tukano du Vaupés plusieurs figures masquées dotées de phallus, qui participent à un même rituel aux côtés d'autres masques incarnant chacun une espèce animale précise. Parmi ces 
dernières, se trouvent de nombreux insectes (mygales, chenilles, larves de scarabée, etc.) dont le dénominateur commun est qu'ils sont tous des proies de guêpes parasitoïdes. À l'inverse des précédents, ces masques dotés de phallus sont désignés sous le générique Dämonen et ne renvoient pas à une espèce particulière, constituant ainsi une exception au regard de l'ensemble des masques (Koch-Grünberg 1995 [1909]: 151, fig. 92). Or, ces personnages phalliques ne sont pas sans partager plusieurs traits avec le Maitre des animaux des Miraña, lui aussi figuré dans le rituel sous cet accoutrement (fig. 5). Notons enfin que, chez Koch-Grünberg, le qualificatif de Dämonen englobe un ensemble d'êtres disparates qui peuplent la forêt sans plus de distinction. Sur les dessins que les Indiens ont réalisés de leurs masques, tous ces " démons » sont dotés soit d'une forte dentition, soit d'un phallus disproportionné, soit, le plus souvent, de ces deux attributs. J'avance donc l'hypothèse suivante : lorsque les sociétés amérindiennes furent pour la première fois confrontées à la figure du diable, il est probable qu'une reconstruction mentale similaire ait pu présider à sa reconnaissance, par assimilation visuelle à l'un de leurs propres personnages «cornus» et phalliques. La dénomination "Yurupari » n'interviendrait que par la suite et serait le fait des missionnaires.

Pour vérifier si cette construction n'est pas le fruit d'une réinterprétation, a posteriori, de la figure du diable par les Indiens de la région, l'une des pistes serait de pouvoir trouver une figuration d'époque précolombienne soit de Yurupari, soit d'une guêpe ou d'un hyménoptère qui posséderait d'un côté les excroissances sur la tête et, de l'autre, les mandibules. Si une telle combinaison devait apparaitre sur des éléments iconographiques précolombiens, elle permettrait de lever l'ambiguité puisque les éléments « diaboliques » n'avaient pas alors encore été introduits et diffusés par la colonisation.

De telles figures n'existent pas pour le Nord-Ouest amazonien, et pour cause: principalement confectionnés pour les rituels, les masques -seuls témoins potentiels de cette iconographie passée- sont non seulement modelés dans des matériaux périssables, mais aussi détruits après chaque rituel. Rappelons que la monstration des flûtes est réservée aux initiés et qu'au moins un type de masque (selon Koch-Grünberg 1995 [1909]: 30), confectionné avec des poils de singe et des cheveux de femmes pubères, était strictement interdit aux femmes, alors que les autres sont entièrement " profanes ».

Il est en revanche possible de trouver de telles illustrations dans les sociétés des Andes précolombiennes. Je fais ici référence à deux pièces de tissu peint, qui possèdent chacune une iconographie renvoyant à la figuration d'une scène mythologique relative à une conjonction astronomique entre un des deux astres majeurs et la constellation d'Orion. Cette dernière est, ici comme chez les Miraña, donnée sous les traits de quatre singes se faisant face symétriquement, en trapèze. Le personnage central est identifié à l'un des astres majeurs, Soleil ou Lune, suivant l'horizon avec lequel il est associé (voir Karadimas 1999, 2003a, 2005). Dans les mythologies miraña où il intervient, le personnage central est une guêpe parasitoïde. 


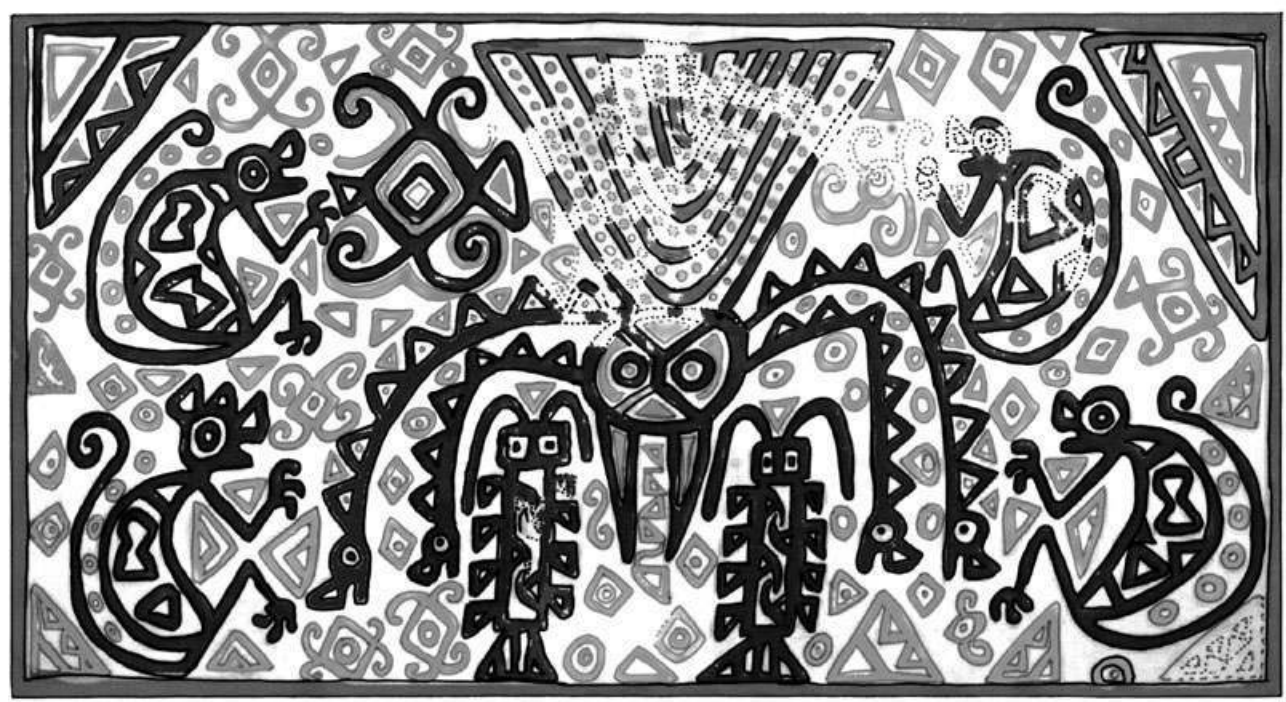

Fig. 6 Textile peint, culture huari (Pérou, côte centrale, 650-800), 117 x 66 cm, d'après illustration sur le site de Rochford \& Messick Fine Arts, 2005.

Dessin de Dimitri Karadimas.

Sur la première pièce de tissu, d'origine huari (fig. 6), les quatre singes sont représentés de façon symétrique, de part et d'autre d'une figure centrale énigmatique, la base la plus courte du trapèze formée par les singes étant dirigée vers le haut. Issue du marché de l'art, cette pièce archéologique n'a pas reçu d'interprétation iconographique. Sur la page Internet où elle est mise en vente, n'apparaît qu'une seule indication concernant la figure centrale, hormis son origine culturelle. Elle représenterait une "grande figure aviaire ", ce qui me semble peu crédible. En effet, si l'on isole chacune des parties qui la composent, il n'y a que les ailes et les deux triangles dépassant de la tête qui pourraient être interprétés comme des ailes et le bec d'un oiseau. Les quatre membres aux extrémités figurées par une tête et agrémentés de motifs en dents de scie ne peuvent pas correspondre aux membres inférieurs d'un volatile (qui n'en possède que deux, et non quatre comme c'est ici le cas) ; leur dédoublement resterait énigmatique.

Plutôt que d'interpréter cette figure suivant l'hypothèse de l'animal imaginaire, je propose d'avoir recours à une lecture figurative et d'opter pour une identification à un hyménoptère. Cette lecture est d'autant plus pertinente que la scène correspond à un thème mythologique (les Quatre Singes) qui s'est maintenu dans le temps, mais non dans l'espace. Les ailes ainsi repliées, les divisions des parties de la tête et les deux yeux formeraient l'image d'une guêpe parasitoïde qui se sert de ses fortes mandibules comme "pince» pour transporter ses proies. Les excroissances triangulaires placées à l'extrémité inférieure de la tête seraient ses mandibules. Les quatre membres émanant de part et d'autre du corps constitueraient quatre des six pattes de l'insecte, les deux autres étant cachées par les ailes repliées (comme c'est le cas chez différentes espèces de pompiles, voir Karadimas 2003b). Les motifs en dents de scie évoquent des dentelures présentes sur les pattes de ces espèces ${ }^{10}$. Il faut surtout reconnaître que la thématique des Quatre Singes associés à une guêpe parasitoïde rejoint celle de la mythologie miraña actuelle dans l'évocation de la conjonction astronomique présentée plus haut. 


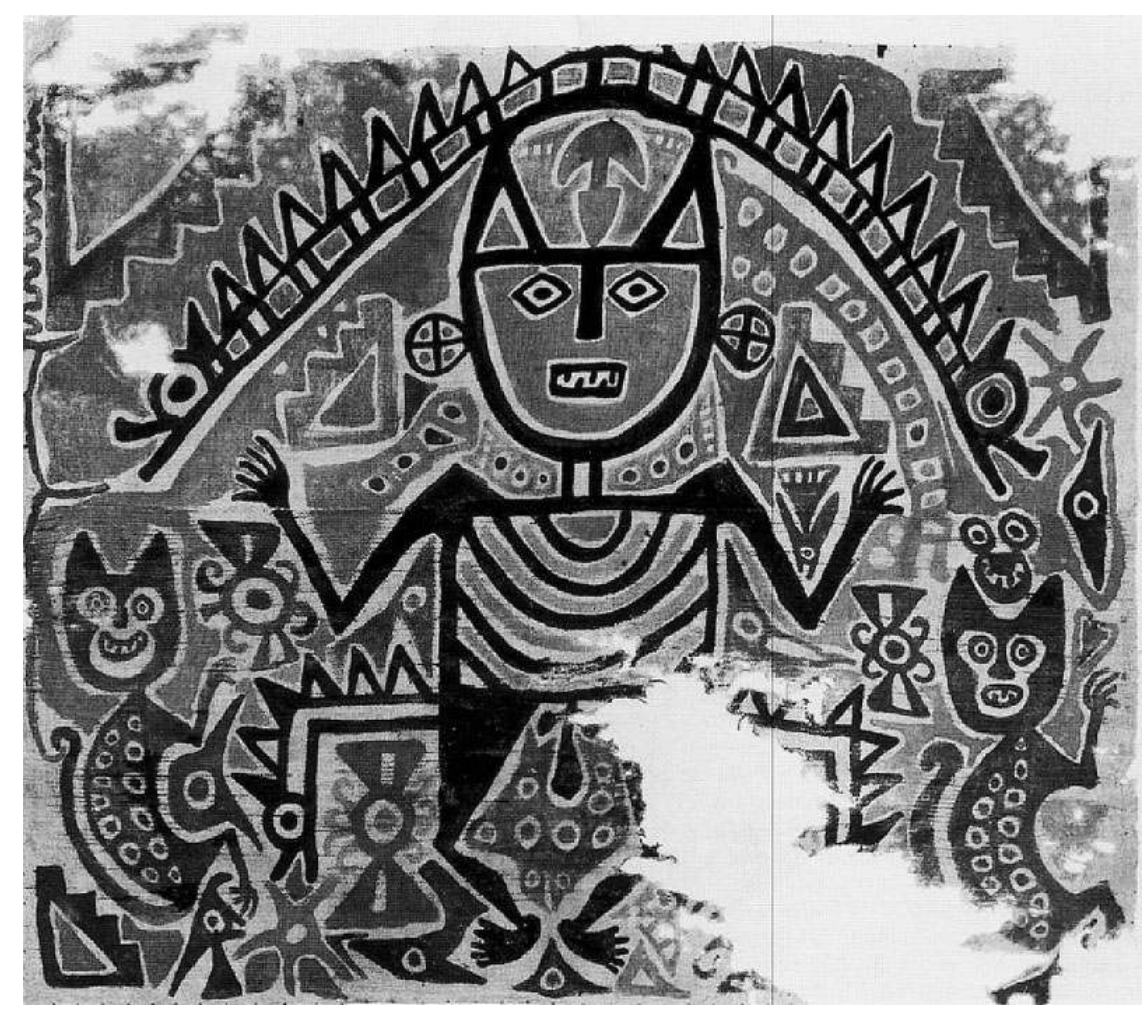

Fig. 7 Textile peint, culture chimú (Pérou, côte nord, VII -XIVe siècles), 132 × 114 cm.

Musée ethnographique de Munich, in Schindler 2001 : fig. 1.

représente une composition similaire à la précédente, bien que distincte quant aux éléments qui la composent (fig. 7). Les quatre singes ne sont plus explicitement figurés, mais remplacés par des cercles concentriques augmentés d'agrégats géométriques. Toujours disposés en trapèze (le quatrième cercle est absent car le tissu est endommagé), ces quatre motifs sont cette fois-ci dirigés avec la base la plus courte vers le bas. Si l'on prend le premier tissu comme point de comparaison, ces cercles inversent le sens de la composition ${ }^{11}$.

Le personnage central retient l'attention car il est la variante anthropomorphe de la figure centrale de la pièce précédente. Il offre un bon exemple d'intermédiaire figuratif entre le tissu d'origine huari et les masques miraña représentant le Maître des animaux. $\mathrm{Si}$, par rapport au textile huari, les ailes ont disparu, l'anthropomorphisation a, comme chez les Miraña, inclus deux éléments supplémentaires. Ont été ajoutés : en premier lieu un pénis pour marquer l'aspect « inséminateur » de l'insecte (selon les mêmes modalités que chez les Miraña), une bouche ${ }^{12}$ ensuite, car les mandibules ont été reléguées sur le haut de la tête, à l'identique du masque miraña. Il suffit de faire effectuer à la figure de la pièce huari un retournement vertical pour que la guêpe, mise de la sorte "debout ", soit anthropomorphisée par l'esprit humain; les deux excroissances triangulaires sont placées en « haut » de la tête... et deviennent des sortes de « cornes ».

31 Les rapprochements que je viens de présenter semblent confirmer mon hypothèse de départ, à savoir que la figuration anthropomorphique d'une guêpe issue de la période précolombienne utilise le même code figuratif que les Miraña: chacun place les mandibules de l'insecte sur le haut -du masque, de la tête- et le dote d'un phallus. Il est donc possible de conclure qu'il ne s'agirait pas, dans le cas de Yurupari et du masque 
miraña, d'une adaptation des figures chrétiennes du diable, dotées d'ailes et de cornes et, pour certaines, d'un caractère sexuel marqué. En effet, les textiles étudiés montrent que de telles configurations existaient avant la période coloniale. Fort des précédentes conclusions, revenons à présent sur les raisons de l'émergence de l'équation établissant une équivalence entre Yurupari et le " diable ", équation qui aurait été construite par les missionnaires. Il semble que face à une démonologie et à une iconographie du diable véhiculée à l'époque par les commerçants, par des croyants, puis surtout par les hommes d'Église, les populations amérindiennes y aient reconnu l'une de leur figure civilisatrice incarnée par une guêpe parasitoïde (nommée ultérieurement "Yurupari » à partir d'un terme tupi qui s'est aujourd'hui cristallisé dans le vocabulaire local). Ainsi, une partie de la chrétienté du xviıe siècle diffusait des descriptions et des iconographies du diable sous les traits d'une figure "cornue", ailée, dotée d'une composante sexuelle -et analemarquée, associée à un bûcher, à un brasier ou aux flammes (celles de l'enfer ${ }^{13}$ ). Le diable était également dépeint en train de dévorer les damnés (tombés dans l'inframonde par une grotte), il prenait parfois la forme d'un serpent (celui du jardin d'Éden et de son fruit défendu) ou d'un dragon, et soufflait dans de larges cornes au bruit assourdissant. Ces récits et ces représentations ne pouvaient que faire apparaître dans l'imaginaire des populations du Nord-Ouestamazonien le personnage de leur propre mythologie puisqu'il partage précisément ces particularités.

La perception d'éléments culturels allogènes génère, par ceux qui les perçoivent, leur réinscription au sein d'éléments culturels déjà connus, afin de leur donner du sens. Le quiproquo interprétatif qui en résulte est toujours en vigueur quant à cette figure majeure du système de croyance des Amérindiens du Nord-Ouest amazonien. Pouvait-il en être autrement ? Chacun ayant « reconnu » chez l'autre ce qu'il pensait être le propre de ses croyances, il aurait alors suffi que l'autre franchisse le pas qui le séparait de la " vérité ». Ce pas, bien sûr, aucune des deux parties n'était prête à le faire, sinon, pour les sociétés amérindiennes, sous la contrainte et l'obligation de la colonisation. Ces dernières, après avoir reconnu leur figure civilisatrice majeure dans le diable chrétien, ont été témoins, en retour, d'une diabolisation (j'ose ici le néologisme de " yuruparisation ")de leur système de croyances et en sont aujourd'hui amenées à trouver dans le dieu chrétien une échappatoire à la diabolisation de leur être, qu'elles ont partiellement intégrée. 


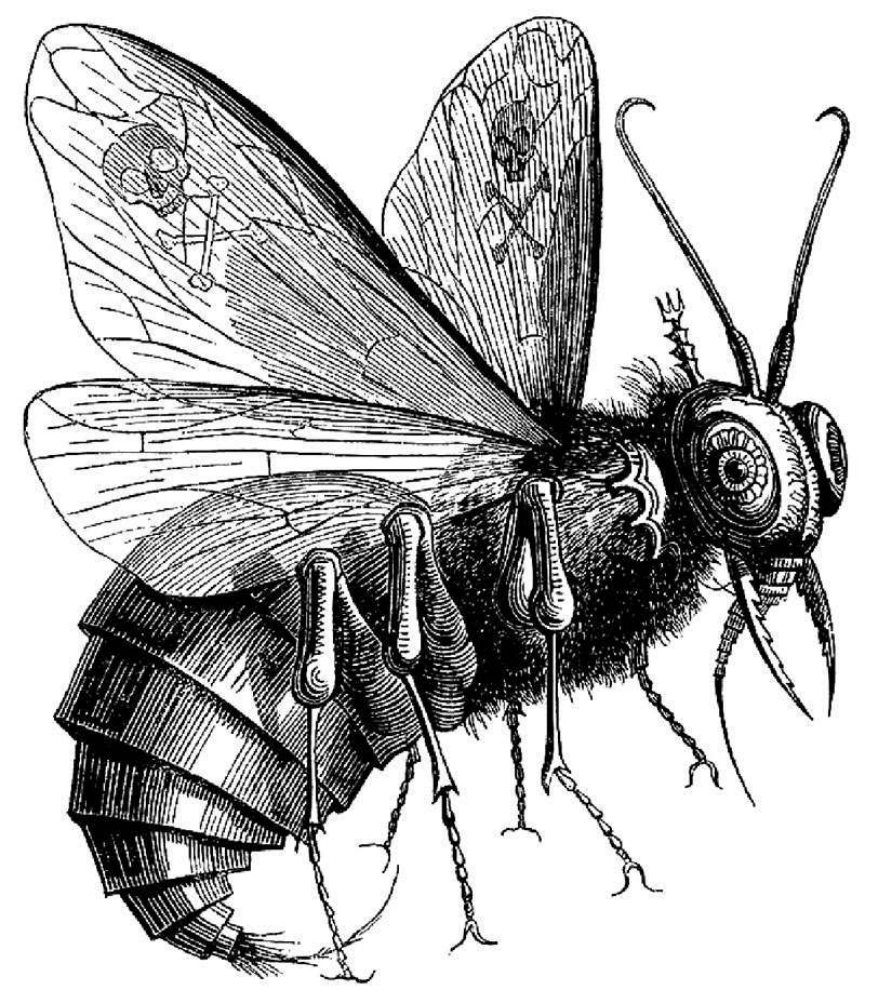

Fig. 8 Belzébuth, ou « Seigneur des mouches ».

In Jacques A. S. Collin de Plancy, Dictionnaire Infernal (Paris, 1863).

Il resterait enfin à s'interroger sur la façon dont les populations du Nord-Ouest amazonien auraient pu réagir, si elles y avaient été confrontées, à l'iconographie de Belzébuth datant du xixe siècle : suite à une interprétation étymologique le qualifiant de «Seigneur des mouches »..., il fut représenté sous la forme d'un hyménoptère rendu hideux (fig. 8), rejoignant ainsi, par bien des aspects, leur propre personnage civilisateur. De la même façon, il serait intéressant de savoir ce que les missionnaires d'aujourd'hui pensent d'une telle figure au regard du personnage des mythologies du Nord-Ouest amazonien que leurs prédécesseurs n'ont pas été en mesure de reconnaître... Diableries, sans doute.

\section{BIBLIOGRAPHIE}

ÅHREM, Kay, CAYón, Luis, ANGULo, Gladys et GARCÍA, Maximiliano (compilateurs)

2004 Etnografía Makuna. Tradiciones, relatos y saberes de la Gente de Agua. Bogotá-Göteborg, Institut colombien d'anthropologie et d'histoire-Acta Universitatis Gothoburgensis.

BUCHILLET, Dominique et al.

1995 Antes o Mundo não existia. Mitologia dos antiguos Desana- $K^{\sim}$ ehíripõrã. São Gabriel da Cachoeira, UNIT/FOIRN. 
1996 A mitologia sagrada dos antigos Desana do grupo Wari Dihputiro Põrã. São Gabriel da Cachoeira, UNIT/FOIRN.

CABRERA BECERRA, Gabriel

2002 La Iglesia en la frontera: misiones católicas en el Vaupés, 1850-1950. Bogotá, Universidad Nacional de Colombia, Sede Leticia.

CÉSARD, Nicolas

2005 « Les épreuves d'insectes en Amazonie », Anthropozoologica 40 (2) : 55-80.

CÉZAR DE CARVALHO, Valeria N.

2006 Les Fils du Tonnerre et l'expansion coloniale : une ethnohistoire du Nord-Ouest amazonien (1750-1889). Paris, École des hautes études en sciences sociales, thèse de doctorat.

FAUSTO, Carlos

2007 «If God were a Jaguar: Cannibalism and Christianity among the Guarani (16th-20th Centuries) ", in Carlos Fausto et Michael J. Heckenberger (éd.), Time and Memory in Indigenous Amazonia: Anthropological Perspectives, vol. I. Gainesville, University of Florida Press : 74-105.

FLECK, Éliane Cristina Deckmann

1999 Sentir, Adoecer e Morrer. Sensibilidade e Devoção no Discurso Missionário Jesuítico do Século XVII. Porto Alegre, Pontifícia Universidade Católica do Rio Grande do Sul, thèse de doctorat.

FRITZ, Samuel

1997 Diarío del padre Fritz, presentado por Hernán Rodriguez Castelo. Quito, XLIX Congreso Internacional de Americanistas.

GOLDMAN, Irving

1963 The Cubeo: Indians of the Northwest Amazon. Urbana, University of Illinois Press.

HUGH-JONES, Stephen

1979 The Palm and the Pleiades. Initiation and Cosmology in Northwest Amazonia. Cambridge, Cambridge University Press.

JOURNET, Nicolas

1995 La Paix des jardins : structure sociale des Indiens curripaco du haut Río Negro (Colombie). Paris, Institut d'ethnologie-musée de l'Homme (« Mémoires de l'Institut d'ethnologie » 31).

KAPFHAMMER, Wolfgang

1992 « Der Yurupari-Komplex in Nordwest-Amazonien », Münchner Amerikanistik Beiträge 28.

KARADIMAS, Dimitri

1999 « La constellation des Quatre Singes. Interprétation ethno-archéoastronomique des motifs de El Carchi-Capulí (Équateur-Colombie) ", Journal de la Société des américanistes, t. LXXXV, Paris : 115-145.

2001 « Singes, raies et étoiles entre les Andes et l'Amazonie. Perspectives comparatives dans l'iconographie amérindienne équatoriale ", Bulletin de la Société suisse des américanistes 64-65 : 83-96. 
2003a « Le masque de la raie. Étude ethno-astronomique de l'iconographie d'un masque rituel miraña », L'Homme 165, numéro spécial « Image et Anthropologie », sous la direction de Carlo Severi : 173-204.

$2003 \mathrm{~b}$ « Dans le corps de mon ennemi. L'hôte parasité chez les insectes comme un modèle de reproduction chez les Miraña d'Amazonie colombienne », in Élisabeth Motte-Florac et Jacqueline Thomas (éd.), Les « Insectes » dans la tradition orale. Paris, Peeters-SELAF n 407 : 487-506.

2005 La Raison du corps. Idéologie du corps et représentations de l'environnement chez les Miraña d'Amazonie colombienne. Paris, Peeters-SELAF (« Langues et sociétés d'Amérique traditionnelle » 10).

À paraître «La métamorphose de Yurupari : flûtes, trompes et reproduction sans femme dans le Nord-Ouest amazonien ", Recherches amérindiennes au Québec (numéro spécial La Sexualité en Amazonie - titre provisoire -, dirigé par Claire Lorrain).

KOCH-GRÜNBERG, Theodor

1995 [1909] Dos Años entre los Indios. Viajes por el Noroeste brasileño 1903-1905. Bogotá, Universidad Nacional de Colombia.

MARTINI, Monica

1990 « Imagen del Diablo en las Reducciones Guaranies », Revista Investigaciones y Ensayos 40 :

175-227.

ORJUELA, Héctor H.

1983 Yuruparí: mito, leyenda y epopeya del Vaupés. Con la traducción de la "Leggenda delli Yurupary " del conde Ermando Stradelli, por Susana N. Salessi. Bogotá, Instituto Caro y Cuervo 3 LXV (Biblioteca de publicaciones del Instituto Caro y Cuervo).

REEVE, Mary Elizabeth

1994 « Regional Interaction in the Western Amazon: The Early Colonial Encounter and the Jesuit Years : 1538-1767 », Ethnohistory 41 (1) : 106-138.

REICHEL-DOLMATOFF, Gerardo

1996 Yuruparí: studies of an Amazonian foundation myth. Cambridge (Massachusetts), Harvard University Press.

SCHINDLER, Helmut

2000-2001 « Imagineria en el Antiguo Perú », in Bulletin de la Société suisse des américanistes 64-65: 69-72.

WRIGHT, Robin M.

1992 « História indígena do Noroeste da Amazônia », in Manuela Carneiro da Cunha (éd.), História dos índios no Brasil. São Paulo, Companhia das Letras-Secretaria Municipal de Cultura : 253-266.

1993 " Pursuing the Spirit. Semantic Construction in Hohodene Kalidzamai Chants for initiation », Amerindia 18.

WRIGHT, Robin M. et HILL, Jonathan

1986 « History, Ritual, and Myth: Nineteenth Century Millenarian Movements in the Northwest Amazon », Ethnohistory 33 (1) : 31-54. 


\section{NOTES}

1. On en trouvera une excellente revue dans le travail de Kapfhammer (1992).

2. Elle date $\mathrm{du} \mathrm{xx}^{\mathrm{e}}$ siècle, et inclut notamment Koch-Grünberg, Goldman, Reichel-Dolmatoff et Hugh-Jones.

3. Buchillet et al. 1995, 1996, Åhrem et al. 2004.

4. Dans sa contribution p. 37 de ce numéro, Goulard suggère la proximité possible des Juri (Yuri) et des Ticuna.

5. On trouvera une bonne analyse des sources ethnohistoriques de cet événement dans la thèse de doctorat de Cézar de Carvalho (2006). N'ayant pris connaissance que très récemment de cette étude, je me réserve la discussion de l'iconographie du masque tariana de cet épisode pour un autre travail.

6. Pour plus de détails sur les ethnies, voir Koch-Grünberg 1995 (1909) et Goldman 1963.

7. Je renvoie le lecteur à un article à paraître (Karadimas à paraître), dans lequel j'examine avec plus de minutie les quatre narrations mythiques proposées par Reichel-Dolmatoff (1996), tout en faisant une comparaison avec la mythologie miraña et avec les données proposées par HughJones (1979). Pour un exposé et une analyse des matériaux strictement miraña, on pourra se référer à mon ouvrage paru en 2005.

8. "Yurupari » traduit également aujourd'hui dans la région non seulement le nom du personnage mythologique, mais aussi tout ce qui est de l'ordre de l'interdit et plus particulièrement la vision des organes sexuels de l'autre sexe par un observateur masculin ou féminin (voir Karadimas 2005).

9. Pour les raisons de son assimilation avec le Maître des animaux, voir Karadimas 2003b.

10. Expliquer pourquoi elles finissent en forme de tête demanderait un autre développement qui dépasse notre propos. Les deux figures qui encadrent la figure centrale - des scolopendres - sont, avec les guêpes pompiles, les seules prédatrices des mygales; leur rôle dans cette composition exigerait, de même, d'étendre notre démonstration.

11. Cette inversion est due à la disposition des étoiles de la constellation, qui se renverse suivant chacun des horizons. Les deux représentations zoomorphiques qui entourent le personnage central sont des compositions empruntant autant au jaguar (pelage ocellé) qu'au singe (visage trilobé) et, dans ce cas, marqué de trois traits, c'est-à-dire faisant référence au singe nocturne Aotus trivirgatus, en l'occurrence l'espèce qui intervient dans la mythologie miraña (pour une discussion de la thématique, voir Karadimas 1999).

12. Rappelons que dans la mythologie le personnage de Yurupari a dû se faire ouvrir une bouche puisqu'il en était dépourvu (cf. supra).

13. Ce rapprochement entre le " brasier » de Yurupari et l'enfer aux yeux des populations du Nord-Ouest amazonien a déjà été proposé par Wright (1993).

\section{RÉSUMÉS}

Yurupari est une des figures emblématiques des sociétés indigènes du Nord-Ouest amazonien. Matérialisé par des flûtes lors des rituels d'initiation masculine, le personnage que celles-ci sont censées incarner reste en partie énigmatique. Les interdits auxquels sont soumis les femmes et 
les non-initiés, le rituel, le mythe... l'ensemble forme un « complexe » qui confine au sacré.

D’abord associé au «malin » ou au "diable » par les missionnaires qui, au XIx siècle, tentaient d'en éradiquer la présence et le rituel, ce complexe a longtemps fait l'objet, dans la littérature associée à cette aire culturelle, d'abondants débats où il intervenait en tant que construction protéiforme proche du monothéisme.

L'article se propose de revenir brièvement sur les éléments composant ce complexe et de les éclairer à l'aide d'une analyse des énoncés mythiques et des iconographies de certains masques associés à l'éthologie des êtres qu'ils incarnent.

La proposition d'analyse se fonde sur l'hypothèse que l'iconographie de la figure du diable véhiculée par les missionnaires aurait été reconnue comme "Yurupari » par les Indiens; la reconnaissance aurait ainsi été l'œuvre des Indiens et non celle des missionnaires. La réflexion porte sur ces regards croisés du donné à voir, ainsi que sur les interdits de montrer et de regarder ; bref, le statut du « regard iconographique » est interrogé.

Yurupari is one of the emblematic figures of the indigenous societies of the North-West Amazonia. Materialised by flutes during the masculine initiation rituals, the character they are supposed to incarnate remains partly enigmatic. The prohibitions to which the women and the non-initiated are submitted, the ritual, the myth... the whole forms a "complex", that adjoins the sacred.

At first associated to the "Satan" or the "Devil" by the missionaries who, in the 19th century, attempted to eradicate its presence and its ritual, this complex has for long been the subject, in the literature associated to this cultural region, of abundant debates in which it appeared in terms of a protean construction close to monotheism.

The article intends to review briefly the different elements composing this complex in order to highlight them from the analysis of the mythic enunciations and the iconographies of a number of masks associated to the ethology of the entities they incarnate.

The proposition of analysis will be based on the hypothesis that the iconography of the figure of the devil conveyed by the missionaries would have been recognised as "Yurupari" by the Indians; the recognition would thus have been due to the Indians and not to the missionaries. The reflection will bear on these crossed points of view of the given to see, and on the prohibitions to show and see; in brief the status of the "iconographic point of view" will be examined.

\section{INDEX}

Mots-clés : Amazonie, Indiens, Yurupari, conversion, missionnaires, diable, perception

Keywords : Amazonia, Indians, missionaries, the Devil

\section{AUTEUR}

\section{DIMITRI KARADIMAS}

CNRS, Laboratoire d'anthropologie sociale, dimitri.karadimas@college-de-france.fr 